\title{
Evaluation of stable and non shattering isabgol cultivar - Gujarat isabgol 4
}

\author{
D G Patel, H B Patel*, P T Patel, H Patel \& A U Amin
}

Seed Spices Research Station, Sardarkrushinagar Dantiwada Agricultural University, Jagudan-382 710, Mahesana, Gujarat.

${ }^{*}$ E-mail: hirenpbg@yahoo.co.in

Received 02 November 2017; Revised 10 April 2018; Accepted 28 April 2018

\begin{abstract}
Isabgol growing area was surveyed during 2009-10 and selected genotypes were evaluated in preliminary evaluation trial (PET) during 2010-13 and in large scale varietal trial (LSVT) during 2013-15. It was found that JI-09-21 recorded better growth and yield characters and yield over check Gujarat Isabgol 3. The JI-09-21 did not shatter much even after water dripping and recommended for cultivation as Gujarat Isabgol 4.
\end{abstract}

Keywords: gujarat isabgol 4, isabgol, new variety, non shattering type

Isabgol (Plantago ovata Forsk) is a short duration, more remunerative and medicinally important crop of arid and semiarid regions. In India, it is largely grown in Gujarat, Rajasthan, Madhya Pradesh and Haryana. The area under Isabgol in India during 2014-15 is 1.09 lakh hectares. The production recorded 72 thousand MT with productivity of $660 \mathrm{~kg} \mathrm{ha}^{-1}$. In Gujarat, area mostly falls in Banaskantha, Kachchh and Patan districts with acreage of nine thousand hectare with production and productivity $5000 \mathrm{MT}$ and $556 \mathrm{~kg} \mathrm{ha}^{-1}$, respectively during 2015-16 (Anonymous 2016a). During the last decade, area and production of isabgol has decreased to the tune of $343 \%$ and $281 \%$, respectively, mainly due to problems of seed shattering. At the time of maturity, unseasonal rain or heavy dew leads to failure of the crop which is the fact for reduction of area under Isabgol (Anonymous 2016b). The objective of the study was to evolve non-shattering isabgol cultivars.
Isabgol growing area of Kachchh in Gujarat was surveyed during 2009-10 and subsequently genotypes were evaluated. First three years (2010-11 to 2012-13) 13 genotypes (JI-09-03, 07, $10,13,15,16,20,21,22,23,24,25$ and 26) with check (Gujarat Isabgol -03) were evaluated in preliminary evaluation - trial (PET). During 2013-14 and 2014-15, nine genotypes (JI-08-02, JI-09-07, 13, 16, 20, 21, 22, 24 and 25) along with check (Gujarat Isabgol -03) was tested. The trials were carried out at three different locations viz., Jagudan, Kholwada and Deesa in a randomized block design with three replications.

The stability analysis of variance and stability parameters viz., linear regression coefficient (bi) and deviation from regression $\left(\mathrm{S}^{2} \mathrm{di}\right)$ of genotype means over environment were computed as suggested by Eberhart \& Russell (1966).

Shattering per cent was computed as suggested by Singh et al. (2005) and Chandra (1967). Five 
plants in each replication were selected at maturity stage. Entire spike were dipped in water and then observed for seed shattering from selected plants. The percentage of seed shattering calculated by using a following formula.

Shattering per cent $=[($ Expected seed yield Seed wt. after threshing) / (Expected seed yield)] $\times 100$

Expected seed yield= H.I. x Sun Dry wt. (kg)/ 100

Considering eight trials at three locations for five years, JI-09-21 was recorded higher $(928 \mathrm{~kg}$ $\mathrm{ha}^{-1}$ ) seed yield against $830 \mathrm{~kg} \mathrm{ha}^{-1}$ of Gujarat Isabgol 3 (GI 3), which was $11.78 \%$ higher than GI-3 (Table 1). The new variety named as
Gujarat Isabgol 4 was having more tillers plant $^{-1}(6.0)$, more spikes plant ${ }^{-1}(22.7)$, seeds spike $^{-1}$ (80.7), higher test weight (1.58 gm) and high swelling factor $\left(11.4 \mathrm{cc} \mathrm{g}^{-1}\right)$ than that of check variety GI-3, due to these yield contributing traits GI-4 is having high seed yield potential (Table 3 ). The similar kind of results also obtained by Prajapati et al. (2011).

Gujarat Isabgol 4 recorded high mean with regression coefficient (bi) near unity and deviation from regression $\left(\mathrm{S}^{2} \mathrm{di}\right)$ around zero for seed yield, indicating GI-4 has average responsiveness and are highly stable over environments (Table 2).

The new culture has compact spike and did not separate easily even after dipping in water. Only $7.25 \%$ seeds shattered after dipping in water.

Table 1. The comparative yield performance $\left(\mathrm{kg} \mathrm{ha}^{-1}\right)$ of JI-09-21 (GI 4) over different locations

\begin{tabular}{|c|c|c|c|c|c|c|c|c|}
\hline \multirow[t]{2}{*}{ Year } & \multirow[t]{2}{*}{ Trial } & \multicolumn{2}{|c|}{ Yield $\left(\mathrm{kg} \mathrm{ha}^{-1}\right)$} & \multirow[t]{2}{*}{ IOC (\%) } & \multirow[t]{2}{*}{ Rank } & \multirow[t]{2}{*}{ S.Em. \pm} & \multirow{2}{*}{$\begin{array}{c}C D \\
(\mathrm{P}<0.05)\end{array}$} & \multirow[t]{2}{*}{ C.V. $\%$} \\
\hline & & JI-09-21 & GI-3 & & & & & \\
\hline \multicolumn{9}{|l|}{ Jagudan } \\
\hline $2010-11$ & PET & 1014 & 894 & 13.42 & $1 / 14$ & 56 & 167 & 10.80 \\
\hline 2011-12 & PET & 1078 & 948 & 13.71 & $1 / 14$ & 49 & 150 & 10.39 \\
\hline $2012-13$ & PET & 907 & 863 & 5.10 & $2 / 14$ & 51 & 155 & 9.47 \\
\hline 2013-14 & LSVT-II & 946 & 874 & 8.24 & $1 / 10$ & 52 & 155 & 11.55 \\
\hline \multirow[t]{2}{*}{ 2014-15 } & LSVT-II & 949 & 812 & 16.87 & $1 / 10$ & 50 & 148 & 11.12 \\
\hline & Mean (5) & 979 & 878 & 11.46 & - & - & - & - \\
\hline \multicolumn{9}{|c|}{ Kholwada } \\
\hline 2013-14 & LSVT-II & 878 & 769 & 14.17 & $1 / 10$ & 52 & 154 & 13.78 \\
\hline \multirow[t]{2}{*}{ 2014-15 } & LSVT-II & $852 *$ & 759 & 12.25 & $1 / 10$ & 31 & 91 & 9.56 \\
\hline & Mean (2) & 865 & 764 & 13.22 & - & - & - & - \\
\hline \multicolumn{9}{|l|}{ Deesa } \\
\hline 2014-15 & LSVT-II & 799 & 722 & 10.66 & $3 / 10$ & 73 & 215 & 14.79 \\
\hline \multicolumn{2}{|c|}{ Overall Mean (8 trials).... } & 928 & 830 & 11.78 & - & - & - & - \\
\hline \multicolumn{2}{|c|}{ Superiority over check.... } & $8 / 8$ & - & - & - & - & - & - \\
\hline
\end{tabular}

Table 2. Stability Analysis for seed yield in Isabgol

\begin{tabular}{lccc}
\hline Variety & Yi (mean seed yield $)\left(\mathrm{kg} \mathrm{plot}^{-1}\right)$ & bi(reg. coeff.) & S $_{\text {di }}$ (mean Sq. dev.) \\
\hline JI -09-21(GI 4) & 0.82 & 0.94 & 0.00 \\
GI-3 (Ch) & 0.42 & 0.80 & 0.00 \\
Mean & 0.39 & - & \\
\hline
\end{tabular}


Table 3. Comparative yield and quality attributes of JI -09-21 (GI 4)

\begin{tabular}{lcc}
\hline Character & \multicolumn{2}{c}{ Mean } \\
\cline { 2 - 3 } & JI -09-21 (GI 4) & GI 3 \\
\hline Days to flowering & 63 & 104 \\
Days to maturity & 102 & 31.3 \\
Plant height (cm) & 31.0 & 5.0 \\
No. of tillers plant ${ }^{-1}$ & 6.0 & 20.2 \\
No. of spikes plant ${ }^{-1}$ & 22.7 & 4.5 \\
Spike length (cm) & 4.7 & 76.4 \\
No. of seeds spike ${ }^{-1}$ & 80.7 & 1.542 \\
1000 grain weight (g) & 1.579 & 9.1 \\
Swelling factor $\left(\mathrm{cc} \mathrm{g}^{-1}\right)$ & 11.4 & \\
\hline
\end{tabular}

Table 4. Effect of water dipping on shattering of isabgol seed

\begin{tabular}{lcccccccc}
\hline Variety & \multicolumn{2}{l}{ Without Dipping } & H.I $\%$ & \multicolumn{3}{c}{ Dipping in Water (Average of } & four sample) \\
\cline { 2 - 5 } & $\begin{array}{c}\text { Dry wt. } \\
(\mathrm{kg})\end{array}$ & $\begin{array}{c}\text { Seed wt. } \\
(\mathrm{kg})\end{array}$ & & & $\begin{array}{c}\text { Dry wt. } \\
\text { (initial) } \\
(\mathrm{kg})\end{array}$ & $\begin{array}{c}\text { Sun dry } \\
\text { wt. } \\
(\mathrm{kg})\end{array}$ & $\begin{array}{c}\text { Seed wt. } \\
\text { after } \\
\text { threshing } \\
(\mathrm{kg})\end{array}$ & $\begin{array}{c}\text { Shattering } \\
\text { of seeds } \\
(\%)\end{array}$ \\
\hline GI 4 (JI-09-21) & 1.027 & 0.175 & 17.03 & & 0.894 & 0.731 & 0.115 & 7.25 \\
GI 3 & 0.959 & 0.158 & 16.47 & 1.038 & 0.811 & 0.037 & 70.07 \\
\hline
\end{tabular}

GI-4 recorded $89.67 \%$ less shattering than that of check variety, which revealed that Gujarat Isabgol 4 was non shattering in habit as compared to Gujarat Isabgol 3 which is prone to high seed shattering. The post dipped seed weight of GI 4,-was $0.115 \mathrm{~kg}$ threshed seeds, which was $96 \%$ higher than that of the post dipped seed yield of GI-3 (0.037 kg threshed seeds) (Table 4). Hence, Gujarat Isabgol 4 recommended for cultivation.

\section{Acknowledgments}

The authors are thankful to Prof. M.M. Patel and Prof. P.R. Patel, Assistant Research Scientist, SDAU, Kholwada and Dr. S.K. Jain, Assistant Research Scientist, SDAU, Deesa, for technical support and to conduct experiment.

\section{References}

Anonymous 2016a District wise area and production of spices crops in Gujarat State.
Directorate of Agriculture, Gujarat State, Gandhinagar.

Anonymous 2016b Spices exports go up by 15 per cent in value. Spices India. A Journal Published by Spices Board, Cochin. 1: 7.

Chandra V 1967 Studies in cultivation of Plantago ovata Forsk. Indian J. Pharm. 29: 331-332.

Prajapati D B, Patel K P \& Ravindrababu Y 2011 Gujarat Isabgol-3 a promising cultivar of Isabgol. Int. J. Forest Crop Improv. 2: 81-84.

Eberhart S A \& Russell W A 1966 Stability parameters for comparing varieties. Crop Sci. 6: 36-40.

Singh R N, Patel K V \& Desai N N 2005 Value addition through processing of Isabgol. In: Tikka S B S, Arha M D, Jaimini S N, Tajane K $\mathrm{R}$ \& Patel N B (Eds.). Abstr. National Symposium on Stress Management. 11-13 April, S. D. Agriculture University, S. K. Nagar, Gujarat (pp.147), Gujarat Society of Genetics \& Plant Breeding, S. K. Nagar. 